1

3

4

6

\title{
Changes in nanomechanical properties of single neuroblastoma cells as a model for oxygen and glucose deprivation (OGD)
}

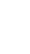

(1)

\author{
Tomasz Zieliński ${ }^{1}$, Joanna Pabijan ${ }^{1}$, Bartłomiej Zapotoczny ${ }^{1}$, Joanna Zemła ${ }^{1}$, Julita \\ Wesołowska ${ }^{2}$, Joanna Pera ${ }^{3}$ and Małgorzata Lekka ${ }^{1 *}$,
}

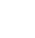

(1)

$$
\begin{aligned}
& { }^{1} \text { Department of Biophysical Microstructures, Institute of Nuclear Physics, Polish } \\
& \text { Academy of Sciences, PL-31342, Krakow, Poland } \\
& { }^{2} \text { Laboratory of in vivo and in vitro imaging, Maj Institute of Pharmacology, Polish } \\
& \text { Academy of Sciences, } 12 \text { Smętna St., PL-31343 Kraków, Poland }
\end{aligned}
$$

\footnotetext{
${ }^{3}$ Department of Neurology, Faculty of Medicine, Jagiellonian University Medical College,
} Botaniczna 3, PL-31503, Kraków, Poland

* Corresponding Author

Malgorzata.lekka@ifj.edu.pl 


\section{Abstract}

The biological processes underlying ischemic stroke, although complex, are better known than those related to biomechanical alterations of single cells. Mechanisms of biomechanical changes and their relations to the molecular processes are crucial for understanding the function and dysfunction of the brain. In our study, we applied atomic force microscopy (AFM) to quantify the alterations in biomechanical properties in neuroblastoma SH-SY5Y cells subjected to oxygen and glucose deprivation (OGD) and reoxygenation $(\mathrm{RO})$. Obtained results reveal several characteristics. Cell viability remained at the same level, regardless of the OGD and RO conditions, but, in parallel,

37 the metabolic activity of cells decreased with OGD duration. 24h RO did not recover the metabolic activity fully. Cells subjected to OGD appeared softer than control cells. Cell softening was strongly present in cells after $1 \mathrm{~h}$ of OGD and, with longer OGD duration and in RO conditions, cells recovered their mechanical properties. Changes in the nanomechanical properties of cells were attributed to the remodelling of actin filaments, which was related to cofilin-based regulation and impaired metabolic activity of cells. The presented study shows the importance of nanomechanics in research on ischemic-

44 related pathological processes such as stroke.

Keywords: cell biomechanics; oxygen and deprivation model; neurodegenerative

47 diseases; stroke; 


\section{Introduction}

51 Ischemic stroke remains one of the leading causes of death, especially in the elderly ${ }^{1}$. It

52 is caused by disrupted blood flow to the brain resulting in oxygen and glucose

53 deficiencies in the cells. The last three decades show significant improvements in acute

54 treatment, resulting in increased life expectancy after treatment and rehabilitation ${ }^{1,2}$.

55 Understanding the stroke at the cellular level can be simulated using an in vitro oxygen-

56 glucose deprivation (OGD) model. The model was widely investigated to study ischemic

57 cell death ${ }^{3}$. In the model, cells or tissue slices are exposed to hypoxic or anoxic

58 conditions and cultured in media deprived of glucose. Not only the effect of OGD is

59 investigated in the model - after changing media and introducing normal oxygen levels,

60 reperfusion can be additionally tested. With long-lasting OGD, the reoxygenation may

61 paradoxically cause additional damage. Ischemia-reperfusion injury is caused by the

62 immediate generation of reactive oxygen species, altered ion transport, and calcium

63 influx ${ }^{4}$. During OGD, rapid remodeling of the actin cytoskeleton was reported to be

64 involved in the blood-brain barrier disruption and affected: endothelial cells ${ }^{5}$, non-

65 neuronal brain cells ${ }^{6}$, and neurons ${ }^{7}$.

66

67

Actin filaments occur in a cell as a meshwork or bundles of parallel fibers abundant, particularly close beneath the cell membrane ${ }^{8,9}$. The continuous control of the balance between polymerization and depolymerization ensures a dynamic equilibrium state, controlling cell architecture, mechanical resistance, and regulating many biological processes $^{10}$. The dynamic of this process is regulated by actin-binding proteins ${ }^{11}$. Cofilin, an actin-depolymerizing factor, was highlighted several times to play a crucial role in actin remodeling in axons ${ }^{7,12,13}$. In ischemia-induced actin disruption, cofilin was 
73 linked with ATP depletion ${ }^{14}$. It has already been reported that cofilin is essential for an

74 early phase of apoptosis ${ }^{15}$ or intracellular contractile force generation ${ }^{16}$. The

75 responsibility of cofilin and its role in various diseases makes it a potential target for

76 potential neuroprotective approaches in the early stages of ischemic brain injury. In

77 particular, the SH-SY5Y human neuroblastoma cell line is used to investigate the OGD

78 model of stroke ${ }^{17}$. The cell line is of human origin, allowing for a better reflection of the

79 induced changes during the stroke. Both non-differentiated and differentiated SH-SY5Y

80 cells have their advantages and drawbacks in the model of neuron cells ${ }^{18}$. In this report,

81 we used undifferentiated cells, which are considered to be most reminiscent of

82 immature neurons ${ }^{19,20}$.

In the present study, we hypothesize that possible involvement of cofilin occurs in

84 the early stages of cytoskeleton remodelling under ischemic conditions. In the initial

85 phase, such remodelling is limited to actin filaments reorganization, which can be quantitatively evaluated using an atomic force microscope $(A F M)^{21}$. This technique is

87 characterized by nanoscale resolution enabled to quantify fine alterations in cells and tissue nanomechanical properties in normal and pathological conditons ${ }^{22-25}$. The

89 changes in mechanical properties have already been shown in undifferentiated SHSY5Y cells in a model of chemically induced neurodegeneration ${ }^{26}$. The results,

91 associated with glutamate-mediated neurodegeneration, showed the increased rigidity

92 of SH-SY5Y cells upon $50 \mathrm{mM} \mathrm{N}$-methyl-D-aspartate (NMDA) treatment. Although the

93 experiment time was limited to $60 \mathrm{~min}$, the maximum rigidity values were obtained after

9420 minutes $^{26}$. Thus, NMDA induced cytoskeletal reorganization. However still, 95 knowledge about the mechanical changes in OGD/reoxygenation (RO) is lacking. Thus, 
96 we analyzed the nanomechanical properties of SH-SY5Y neuroblastoma cells exposed

97 to oxygen and glucose deprivation, mimicking ischemic conditions. Following our

98 previous studies on the effect of anti-tumor drugs on prostate cancer cells ${ }^{27}$, two

99 indentations were applied, i.e., shallow $(400 \mathrm{~nm})$ and deep $(1200 \mathrm{~nm})$ ones. The

100 shallow indentation reveals mechanical properties dominated by actin filaments, while

101 the deep indentation may contain the additional contribution from deeper parts of the

102 cells like the microtubular network and cell nucleus ${ }^{27,28}$. The studies were accompanied

103 by evaluating the cofilin and phosphorylated cofilin expression levels, visualization of

104 actin filaments organization quantified using morphometric parameters, and metabolic

105 activity of SH-SY5Y cells subjected to OGD. Measurements were conducted directly

106 after OGD to study the magnitude of the induced changes and after $24 \mathrm{~h}$ of

107 reoxygenation to model reperfusion and to evaluate the reversibility of these changes.

\section{Results}

110 Viability of SH-SY5Y cells under OGD

111 To assess the effect of OGD exposure $\left(5 \% \mathrm{CO}_{2}, 0.1 \% \mathrm{O}_{2}\right)$ on neuroblastoma SH-SY5Y

112 cells, we exposed SH-SY5Y human neuroblastoma cells to OGD for 1, 3, and 12 hours, 113 followed by 24 hour-RO. (Fig. 1). 


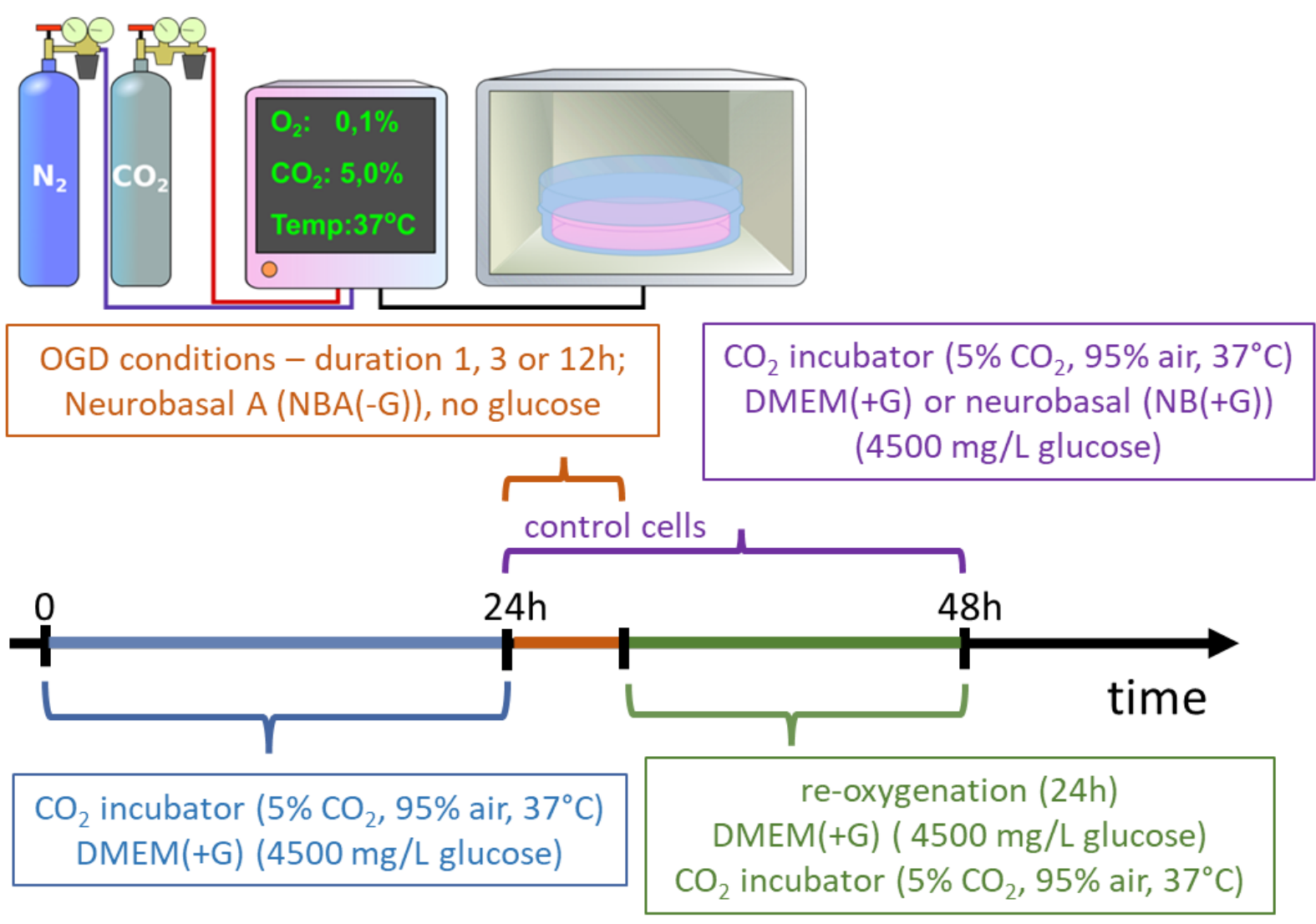

115 Figure 1. (a) A scheme showing three steps of sequential OGD applied to living SH116 SY5Y cells. Firstly, cells were cultured for 24 hours after seeding in 5\% CO2, 95\% 117 atmosphere $\left(37^{\circ} \mathrm{C}\right)$ in a DMEM with $4500 \mathrm{mg} / \mathrm{ml}$ of glucose $(\mathrm{DMEM}(+\mathrm{G}))$. They refer 118 here as control cells. Next, the medium was exchanged to $\operatorname{NBA}(-\mathrm{G})$, and cells were 119 placed in a table $\mathrm{CO}_{2}$ incubator for $1 \mathrm{~h}, 3 \mathrm{~h}$, or $12 \mathrm{~h}$ at $0.1 \% \mathrm{O}_{2}$ (referred to as OGD conditions and OGD cells). Finally, OGD cells were rinsed with a $\operatorname{DMEM}(+\mathrm{G})$ in the 121 atmosphere of $5 \% \mathrm{CO}_{2}$ and $95 \%$ air (reoxygenation conditions, , in addition, non-OGD 122 cells were kept in $\operatorname{DMEM}(+\mathrm{G})$ ). (b) Phase-contrast image showing the morphology of 123 neuroblastoma SH-SY5Y cells cultured for $24 \mathrm{~h}$ in $\mathrm{NB}(+\mathrm{G})$, as it induced differentiation 124 resulting in a neuron-like morphology with numerous, fine protrusions (neurite-like 125 structures). Scale bar $-50 \mu \mathrm{m}$. 
127 We compare four groups of data, namely, control (C, measurements were conducted in 128 neurobasal medium, which contained $4500 \mathrm{mg} / \mathrm{L}$ of glucose, referred here as $\mathrm{NB}(+\mathrm{G}))$,

129 OGD cells (in neurobasal A medium without glucose, NBA(-G)), reoxygenated OGD 130 cells (DMEM, which contained $4500 \mathrm{mg} / \mathrm{L}$ of glucose, DMEM(+G)) and additional control 131 (i.e. non-OGD) cells kept in $\operatorname{DMEM}(+\mathrm{G})$ for the same time as re-oxygenated (RO) OGD 132 cells.

133 We started with the assessments of metabolic activity (using MTS assay; reduction of 134 tetrazolium; impaired $\mathrm{NAD}(\mathrm{P}) \mathrm{H}$ metabolism ${ }^{29}$ ) and cell viability (using LDH assay; 135 lactate dehydrogenase release to culture media, membrane damage ${ }^{30}$ ) that were 136 applied to samples collected directly after OGD and after $24 \mathrm{~h}$ of reoxygenation. The 137 results show that cell metabolism and viability depended on OGD duration (Fig. 2). 138 Moreover, the induced changes are still present in cells after reoxygenation. 


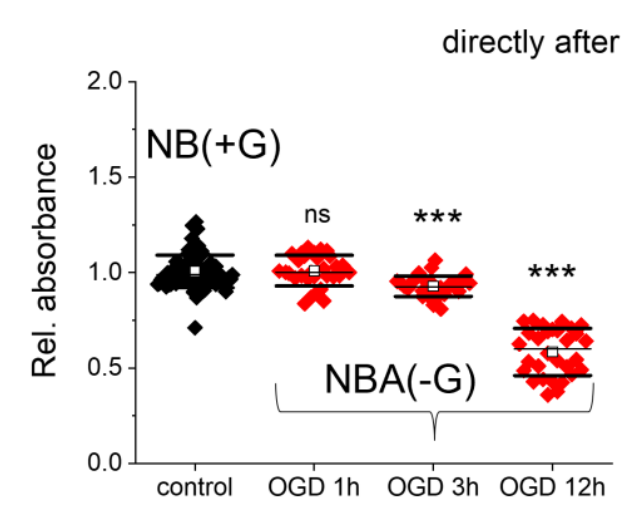

141

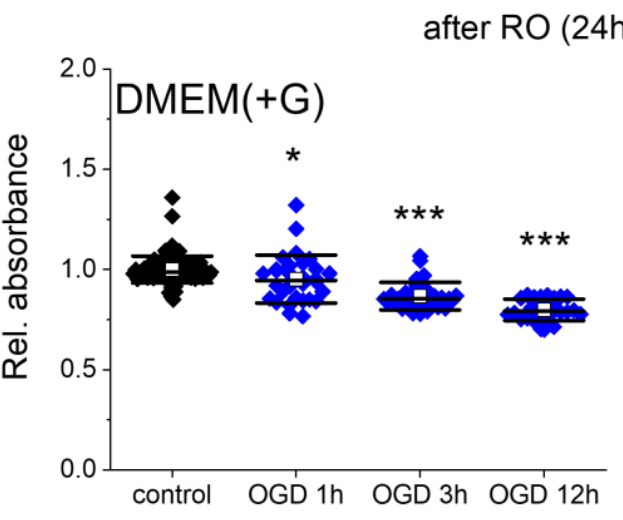

b)

directly after OGD
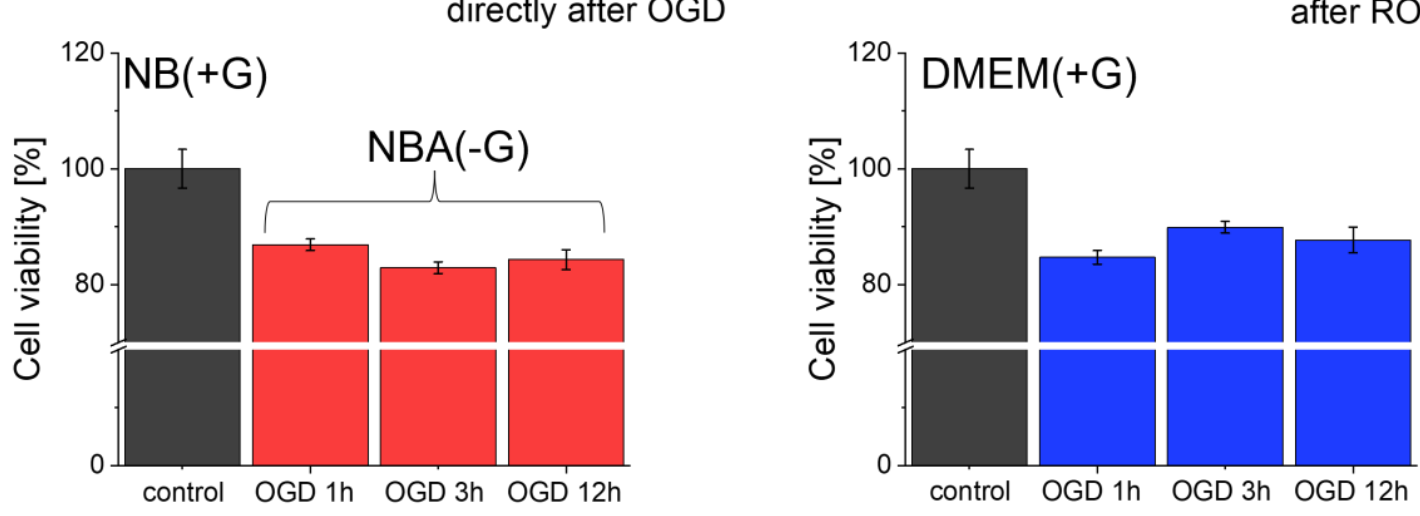

d)

143 Figure 2. Metabolic level and viability of SH-SY5Y neuroblastoma cells assessed by 144 MTS (a,b) and LDH (c,d) assays, directly after OGD (a,c) and after 24h of 145 reoxygenation $(b, d)$. Each dot denotes a single readout from the ELISA reader. $(a, b) A$ 146 mean (open circle), median (black line), standard deviation (SD, box size) were 147 determined from data gathered from 3 independent repetitions. (c,d) Columns represent 148 a mean value from 12 ELISA readouts ( $n=3$ independent repetitions). Relative 149 absorbance was normalized to values obtained for the control samples. Statistical 150 significance: $n s-$ not statistically significant, $p>0.05,{ }^{*} p<0.05,{ }^{* \star *} p<0.001$. 
152 We tested how $1 \mathrm{~h}, 3 \mathrm{~h}$, and $12 \mathrm{~h}$ of OGD affect cell metabolism of SH-SY5Y cells

153 (Fig. 2ab). The MTS tetrazolium is reduced by cells to formazan soluble in the culture

154 medium. Such conversion is presumably accomplished by NADPH or NADH produced

155 by dehydrogenase enzymes in metabolically active cells ${ }^{29,31}$. Thus, lower absorbance in

156 comparison with control cells denotes the lower metabolic activity of cells. Our results

157 show a significant reduction in formazan conversion after $3 \mathrm{~h}$ and $12 \mathrm{~h}$. After one hour of

158 the cell exposure to OGD, the metabolic activity level was similar to that of control cells,

159 and no significant difference was identified ( $p=0.262$; Fig. 2a). However, we do 160 observe changes in reoxygenation for all three groups of cells subjected to OGD. The

161 MTS-based cell viability assessed directly after OGD experiments dropped by about

$1627.1 \%(p<0.001)$ and $41.5 \%(p<0.001)$ after $3 \mathrm{~h}$ and $12 \mathrm{~h}$ of cell exposure to OGD,

163 respectively (Fig. $\mathbf{2 b}$ ). In parallel, we checked membrane integrity by LDH assay related

164 to the number of viable cells. A drop of about $13 \%-17 \%$ was observed for cells after

165 OGD (Fig. 2c). A similar drop in the number of viable cells was observed for cells kept

166 for 24 h in RO. A drop between 12\%-15\% was obtained (Fig. 2d).

167 We expect that cells being damaged by OGD will recover their ability to proliferate ${ }^{32}$.

168 Therefore, both MTS and LdH assays have also been applied to cells cultured in reoxygenation conditions. MTS results revealed significant changes in all groups of cells

170 (Fig. 2b,d). The most significant drop in cell metabolic activity was observed for cells 171 subjected to OGD for $12 \mathrm{~h}$, while a small change, but still statistically significant, was

172 recorded for cells after $1 \mathrm{~h}$ of OGD. Interestingly, the level of LdH remained at a similar

173 level for both control and treated (OGD, RO) cells (Fig. 2c,d), indicating no correlation

174 between cell viability and metabolic activity of cells, regardless of their treatment. 
175 These results show that only metabolic activity was affected after prolonged OGD.

176 Lower metabolic activity was not related to the number of viable cells (or more precisely

177 to the impaired membrane integrity). No new cells were dying after 24h OGD, but their

178 metabolism activity was altered after OGD.

179

180 The effect of different OGD duration on mechanical properties of SH-SY5Y cells

181 To assess whether the altered metabolic activity is related to nanomechanical

182 properties of SHSY\%Y cells, AFM working in a force spectroscopy mode was employed

183 to conduct the measurements over a nuclear region of the cell (to avoid the influence of

184 stiff substrates $^{33}$ ). The nanomechanical properties were quantified by Young's (elastic)

185 modulus calculated by applying Hertz-Sneddon contact mechanics ${ }^{22,34}$, assuming that

186 a cone can approximate the shape of the probing pyramidal tip (Fig. 3). 

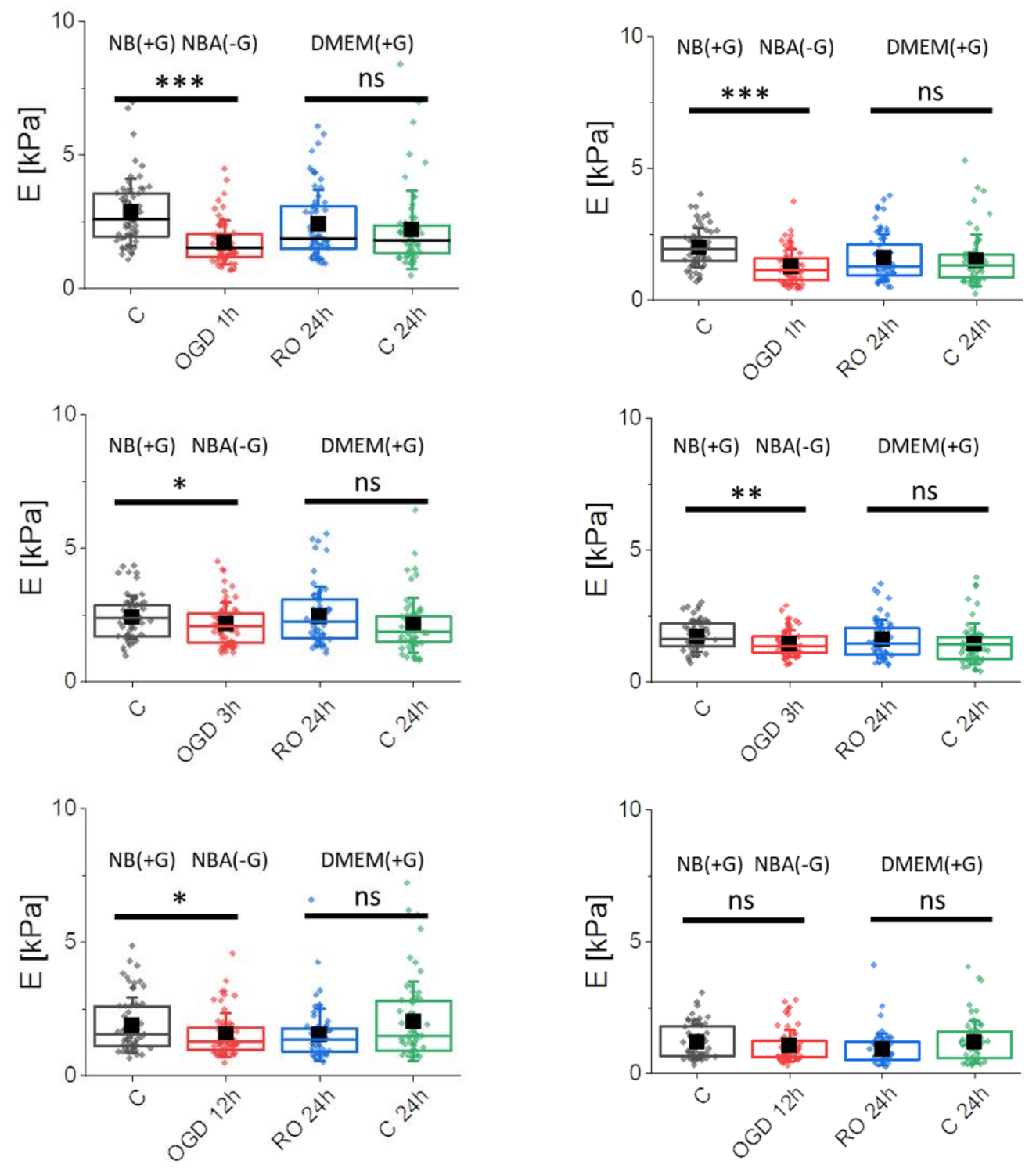

187

a)

b)

Figure 3. Nanomechanical properties of SH-SY5Y neuroblastoma cells after OGD treatment, quantified by the apparent Young's modulus calculated for the indentation

190 depth of $400 \mathrm{~nm}$ (a) and $1200 \mathrm{~nm}$ (b). Four groups of cells were compared: control (C, $191 \mathrm{NB}(+\mathrm{G})$ ), OGD cells (OGD 1h, 3h, or 12h, NBA(-G)), reoxygenated OGD cells (RO 24h, 192 DMEM $(+G)$ ), and control, non-OGD cells (C 24h) kept in DMEM(+G) for the same time 193 as reoxygenated OGD cells. Box plots represent a median (black line), a mean (solid 194 square), standard deviation (whiskers), and $25 \%$ and $75 \%$ percentiles (box) from $n=60$ 
195 196

197

198

199

200

201

202

203

204

205

206

207

208

209

210

211

212

213

214

215

216

217

218

cells. Statistical significance: ns - not statistically significant $(p>0.05),{ }^{*} p<0.05$, ${ }^{\star *} p<0.01,{ }^{* * *} p<0.001$.

Young's modulus was calculated at the shallow and deep indentations, i.e., $400 \mathrm{~nm}$ and $1200 \mathrm{~nm}$, respectively. Alongside the already published data $22,28,35,36$, the nanomechanical response of cells measured at shallow indentation $(400 \mathrm{~nm})$ reflects mainly the mechanics of the actin cortex. Thus, any alteration in cell mechanics can be related to the remodelling of actin filaments underlying beneath the cell membrane. AFM can measure only cells attached to the underlying surface; thus, to a certain extent, the mechanical properties of cells reflect the mechanics of cells resistant to unfavorable conditions. Cells heavily affected by OGD detached from the surface were not accessible for the AFM measurements. Still, in our study, mean values of Young's modulus of OGD-treated cells significantly dropped by about $39.2 \%(p<0.001), 10.7 \%$ $(p=0.045)$, and $19.4 \%(p=0.042)$ for $1 \mathrm{~h}, 3 \mathrm{~h}$, and $12 \mathrm{~h}$ OGD in relation to control cells, respectively (Fig. 2a). Reoxygenation recovers the nanomechanical properties of cells close to values obtained for control, non-OGD cells kept in DMEM(+G) for 24 h (same duration as reoxygenation). The elastic moduli are $2.40 \pm 1.31 \mathrm{kPa}$ versus $2.22 \pm 1.46$ $\mathrm{kPa}(p=0.401), 2.46 \pm 1.09 \mathrm{kPa}$ versus $2.12 \pm 1.03 \mathrm{kPa}(p=0.084)$, and $1.55 \pm 0.98$ $\mathrm{kPa}$ versus $1.05 \pm 1.48 \mathrm{kPa}(p=0.110)$ for OGD-treated and non-treated cells, correspondingly. Thus, we can conclude that the recovery of the actin cortex occurs independently of the OGD duration. The largest changes were observed in cells after $1 \mathrm{~h}$ OGD, but simultaneously, $24 \mathrm{~h}$ of reoxygenation allowed cells to almost fully recover their mechanics. Longer OGD (3h and $12 \mathrm{~h}$ ) resulted in smaller mechanical changes than those observed after 1h of OGD. 
The analysis of deeper indentations (like here $1200 \mathrm{~nm}$ ) can evaluate the combined contributions of the actin cytoskeleton and other structural components of

221 cells such as microtubules or cell nuclei. Mechanics of cells measured directly after

222 OGD shows a significant drop after 1h and 3h. The apparent Young's modulus dropped by $35.5 \%(p>0.001)$ and $16.8 \%(p=0.007)$, respectively (Fig. $\mathbf{2 b})$. The OGD-induced

224 mechanical changes were statistically insignificant after $12 \mathrm{~h}$ of cell exposure to such 225 conditions $(p=0.188)$. A similar level of changes suggests a weaker contribution of

226 other cellular structures as compared to the actin cytoskeleton. In reoxygenated cells, 227 changes in mechanical properties of OGD and non-OGD cells were statistically 228 insignificant, showing a lack of mechanical contributions from deeper cellular layers.

229 These results demonstrate that the mechanical response mainly contains the dominant 230 contribution from the actin cytoskeleton.

\section{Organization of actin cytoskeleton in OGD-treated SH-SY5Y cells}

233 Phase-contrast images collected prior to the AFM measurements did not show any

234 particular changes in the macroscopic morphology. OGD treated cells reveal similar 235 spindle and neuron-like morphology as control, non-treated cells, regardless of the OGD 236 duration. As changes in cell mechanics are typically related to the organization of actin 237 filaments, the confocal images with fluorescently labelled F-actin and cell nucleus were analyzed (Fig. 4). 

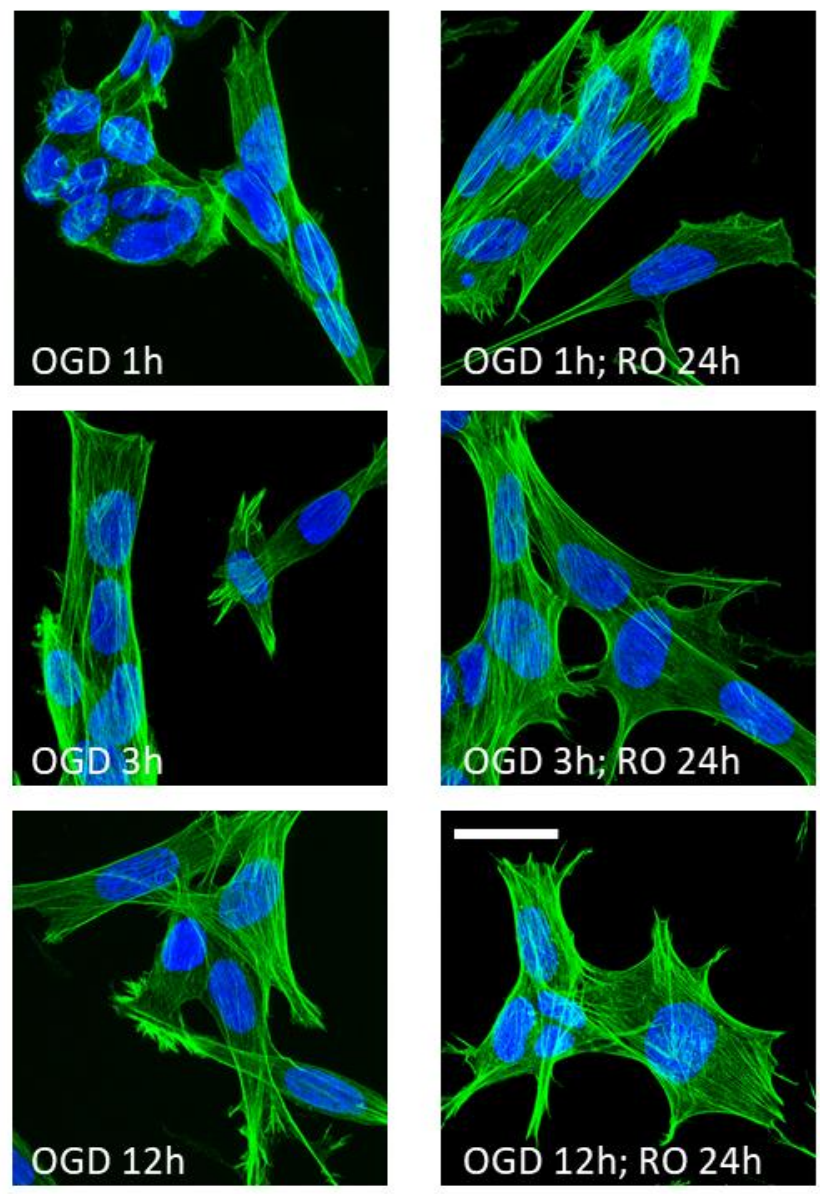

240 Figure 4. Confocal images of the actin cytoskeleton in OGD-treated and reoxygenated 241 cells. Staining: actin filaments - phalloidin conjugated with Alexa Fluor 488, cell nuclei 242 Hoechst 33342; scale bar $25 \mu \mathrm{m}$.

244 In control and OGD treated cells, the organization of actin cytoskeleton was very similar, 245 showing nicely actin bundles spanning over the whole cell. The only exception was cells 246 visualized directly after 1h OGD, where cells change their morphology from a widely 247 spread to a packed one (Fig. 4). This is consistent with the mechanical results showing 248 the largest drop in the apparent Young's (elastic) modulus. The organization of actin 249 filaments in cells undergoing longer OGD treatment ( $3 \mathrm{~h}$ and $12 \mathrm{~h}$ ) was barely visible, 250 supporting weak changes in nanomechanical properties. Altogether, these results 
251 support the conclusion that the nanomechanical properties of cells are dominated by

252 actin filament organization.

253

\section{Cell spreading as a measure of cell attachment}

255 In our further steps, we performed a deeper analysis of the shape of individual cells

256 using images recorded by epi-fluorescent microscopy (Fig. 5).
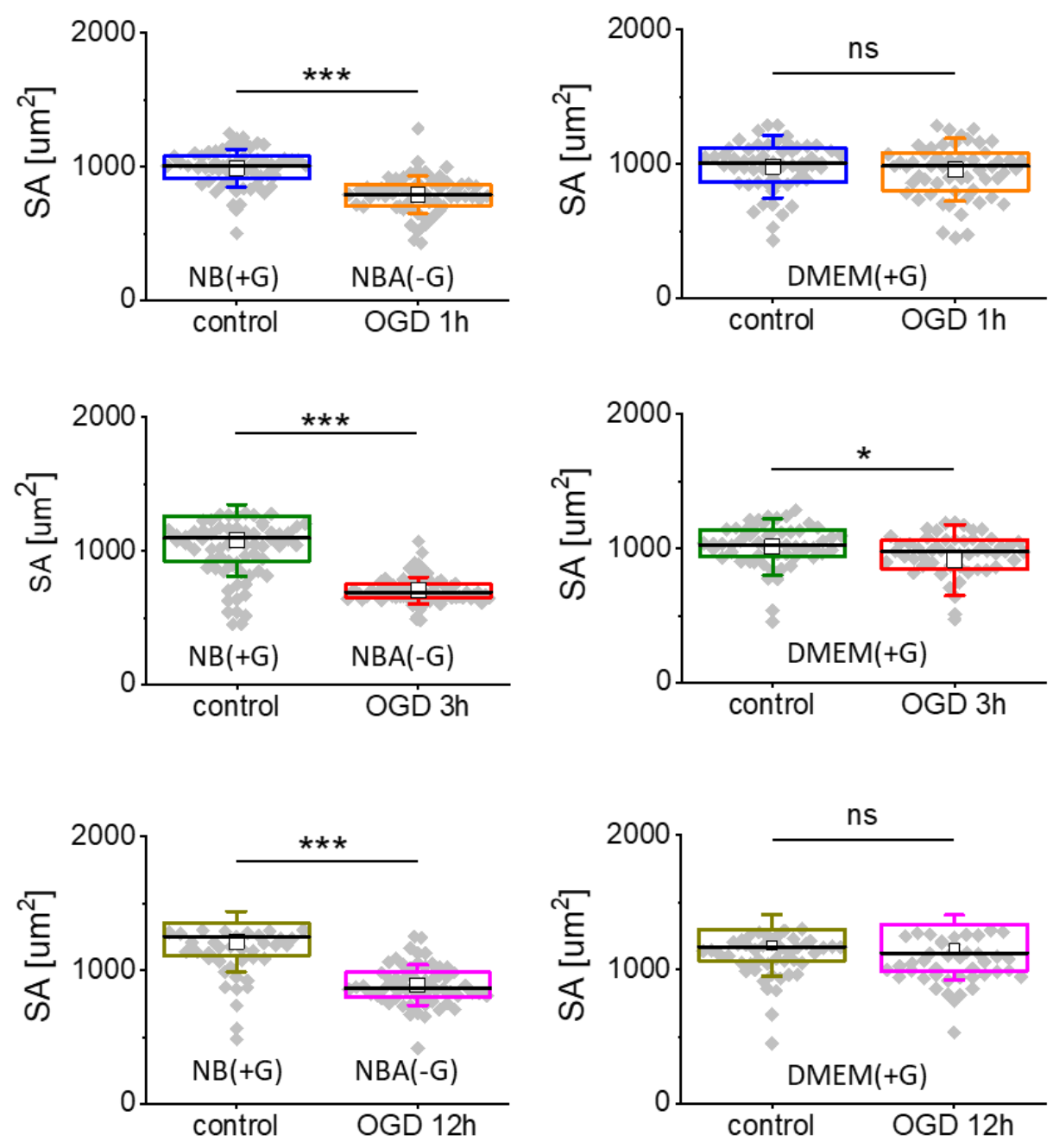

258 Figure 5. Spreading area (SA) of cells after OGD (a) and reoxygenation (b). Each dot 259 denotes an average surface area of individual cells. Boxplot represents basic statistical 260 parameters (mean, median, standard deviation, and $25 \%$ and $75 \%$ percentiles from 
$261 \mathrm{n}=60$ fluorescent images). Statistical significance: $n s$ - not statistically significant $262(p>0.05),{ }^{*} p<0.05,{ }^{* * *} p<0.001$.

264 The results revealed that OGD-treated cells have different surface areas indicating 265 impairments in their spreading on the surface (Fig. 5a). The smaller the surface area, 266 the worse their attachments/adhesion to the surface is. The weak attachment of cells to 267 the underlying surface was recovered after allowing cells to grow in reoxygenation 268 conditions (Fig. 5b). The largest change in spreading area was observed for OGD269 treated cells, while during reoxygenation, cells return to the surface area of control, non-

270 treated cells. These results indicate that the spreading of cells involves the remodelling 271 of actin filaments, which in our case is strongly related to the OGD treatment of SH272 SY5Y cells.

273

274 The shrinking of the cells is confirmed by the nucleus to cell ratio.

275 Changes in cell surface area and lack of strong reorganization of the actin filaments 276 suggest different mechanisms inducing alterations in nanomechanical properties of 277 OGD-treated cells, such as changes in cell volume. A ratio between cell surface area $278(C)$ and cell nucleus $(M)$ can quantify the latter (Fig. 6). 

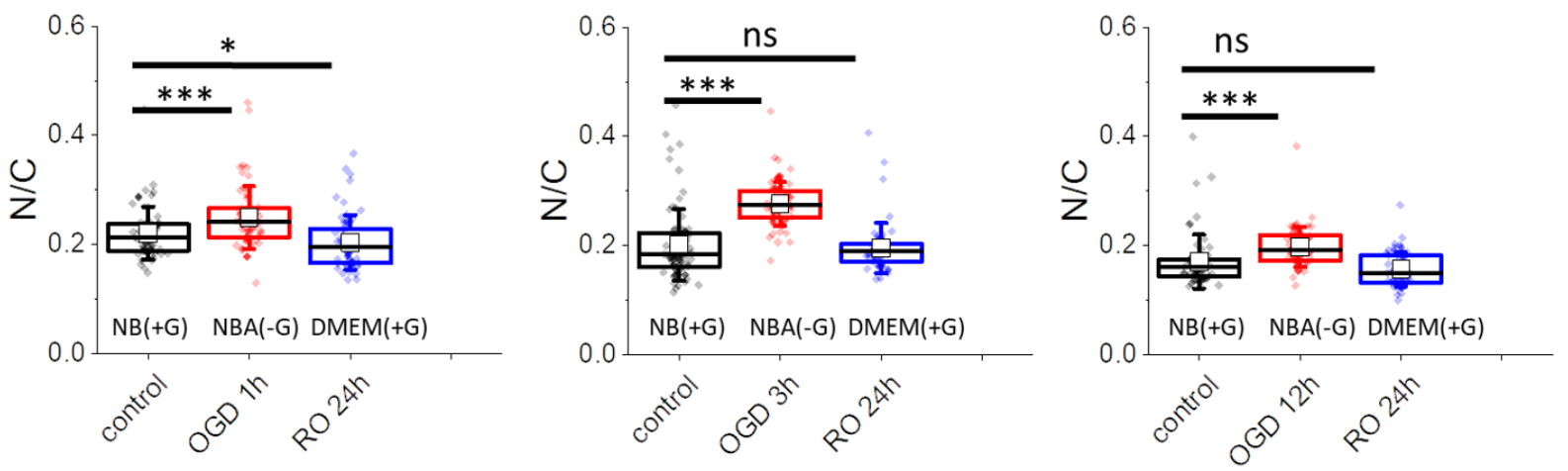

282

Figure 6. Nucleus to cytoplasm $(N / C)$ ratio of cells after OGD treatment and 283 reoxygenation. Each dot denotes an average value of individual cells. Boxplot 284 represents basic statistical parameters, i.e., mean (open square), median (line), and standard deviation, from $n=60$ cells. Statistical significance: $n s$ - not statistically significant ${ }^{*} p<0.05,{ }^{* *} p<0.001$.

The $N / C$ value close to 1 indicates the dominant contribution of a cell nucleus in the surface area value, while its value close to 0 indicates a significant contribution from the cytoplasm.

The results show that the $N / C$ ratio increases in cells visualized directly after OGD for all 292 three tested timepoints and reaches the level of control cells after $24 \mathrm{~h}$ of cell reoxygenation in all groups. Rought estimation of cell height from the cross-section of

294 confocal images (from Fig. 4), shows that the height of the cell in the central area is $7.3 \mu \mathrm{m} \pm 1.4 \mu \mathrm{m}(n=14$ cells $), 8.3 \mu \mathrm{m} \pm 2.0 \mu \mathrm{m}(n=11) 10.9 \mu \mathrm{m} \pm 2.8 \mu \mathrm{m}(n=10)$ for

296 cells after $1 \mathrm{~h}, 3 \mathrm{~h}$, and $12 \mathrm{~h}$ OGD, respectively. Altogether, these results show that the 297 height of cells increases during OGD treatment. 


\section{Cofilin level in OGD-treated SH-SY5Y cells}

301 Cofilin is an actin regulating protein that quickly responds to various cell processes. Alterations in calcium ions, reactive oxygen species, ATP, or $\mathrm{pH}$ will result in quick dephosphorylation (activation) of cofilin ${ }^{37}$. Cofilin severs actin filaments but does not enhance actin depolymerization rate ${ }^{38}$. Instead, it creates new nucleation centres allowing for quick branching, polymerization, and depolymerization in a concentrationOGD treated cells are presented in Fig. $\mathbf{7 .}$

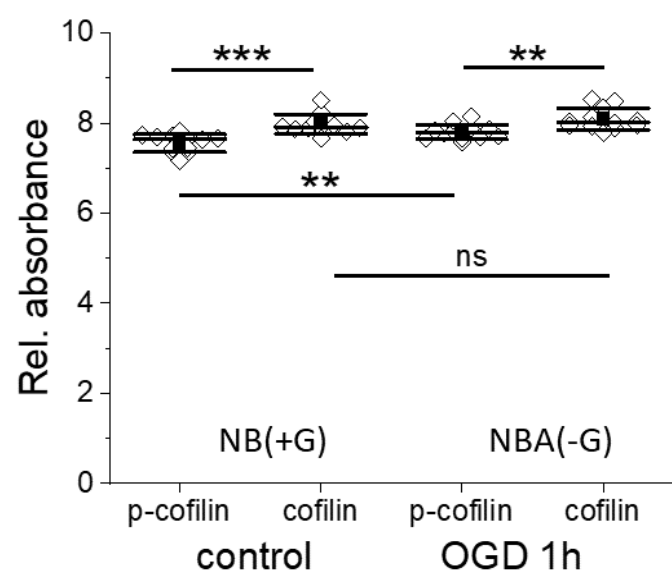

308 a)
OGD $1 \mathrm{~h}$
b)

control

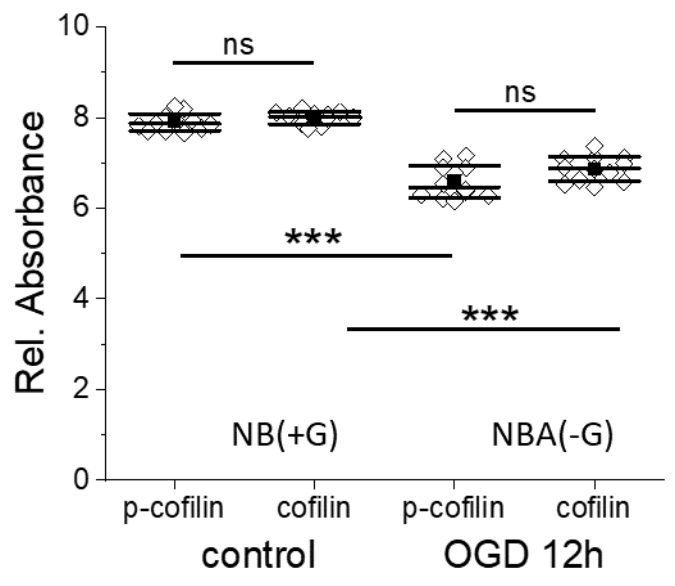

Figure 7. Cofilin and p-cofilin (phosphorylated cofilin) expression level in SH-SY5Y cells upon 1h (a) and 12h (b) OGD. Control cells were kept in $\mathrm{NB}(+\mathrm{G})$, while OGD cells were kept in NBA(-G). A mean (black square), median (black middle line), standard deviation

312 (SD, outside black lines) were determined from data gathered from 3 independent $\left.{ }^{* * *} p<0.001\right)$. 
316 We observed the difference in the cofilin and p-cofilin levels in control cells that could be

317 linked with a lower glucose level observed by proliferating cells in a given volume during

318 a certain culture time ${ }^{40}$. When cofilin/p-cofilin was assessed in control cells

319 simultaneously as cells after $1 \mathrm{~h}$ of OGD, the concentration of cofilin was about $5 \%$

320 higher than $p$-cofilin $(p<0.001)$. In 1 h OGD treated cells, the concentration of cofilin

321 was only $3.5 \%$ larger than $p$-cofilin $(p<0.002)$. In cells exposed to longer OGD

322 duration, the expression level of cofilin in relation to $\mathrm{p}$-cofilin vanishes (the same protein

323 level was observed in cells after $3 \mathrm{~h}$ and 12h OGD (Fig. 7b shows results of cofili/p-

324 cofilin expression in SH-SY5Y cells after 12h OGD). Next, we compare the differences

325 between the expression level of cofilins (or p-cofilins) for control and OGD treated cells.

326 The determined $p$-values were (i) $p=0.006$ ( $p$-cofilin, between control and OGD (1h)

327 cells) and $p=0.198$ (cofilin, between control and OGD (1h) cells); (ii) $p<0.0001$

328 (between control and OGD (3h) cells, regardless of the cofilin status); (iii) $p<0.0001$

329 (between control and OGD (3h) cells, regardless of the cofilin status). These results

330 show that the ratio between cofilin/p-cofilin changes significantly during $1 \mathrm{~h}$ OGD and

331 vanish for a longer duration of OGD. Interestingly, the expression level of cofilin and p-

332 cofilin in OGD cells decreases with OGD duration. Only, the expression level of $p$-cofilin

333 changed.

\section{Discussion}

336 Oxygen and glucose deprivation (OGD) is commonly used to study cerebral ischemic 337 stroke. It mimics the process of a sudden disruption of blood flow to the brain. The lack of blood supply leads to decreased oxygen and glucose levels in the brain. The induced 
339 injuries activate various biochemical processes such as perturbation of calcium

340 homeostasis ${ }^{41}$, malfunction of endoplasmic reticulum and mitochondria ${ }^{42}$, increased

341 level of oxidative stress linked with DNA damage ${ }^{43}$. They directly affect cell morphology,

342 which suggests changes in mechanical properties of OGD treated cells. In our work,

343 AFM was applied to probe nanomechanical properties at the cellular level.

344 Nanomechanics has already been reported to be altered during stroke ${ }^{44}$. The results

345 have shown that tissue mechanics changes within the region affected by stroke and,

346 also, at a distance from the stroke site ${ }^{44}$. AFM has shown the alterations in mechanical

347 properties of the brain region severely affected by ischemia. Neuronal cells are

348 mechanosensitive and highly responsive to altered mechanics of the surrounding

349 environment ${ }^{45}$. Thus, changes in the mechanical properties of ischemic tissue denote

350 also changes in the functioning of neuronal cells.

Our study focused on the nanomechanical properties of SH-SY5Y cells subjected

352 to OGD of different duration followed by 24-hour reoxygenation. AFM-based elasticity

353 measurements were conducted at the shallow and deep indentations, which enabled us

354 to quantify the changes occurring mainly in the network of actin filaments. A drop of

355 Young's modulus, a measure of cell deformability ${ }^{22}$, observed in cells subjected to

356 OGD, suggests the reorganization of the cell cytoskeleton at the layer composed of the

357 actin filaments. The most significant drop of Young's modulus was observed in SH-

358 SY5Y cells measured directly after OGD. When cells were allowed to grow in fully 359 reoxygenated conditions, their elastic properties returned to the level of control cells.

360 The cell metabolic activity (MTS assay) and cell viability (LDH assay) showed that the 361 number of alive cells remained within $83-88 \%$ for both control and OGD treated cells. 
362 However, the metabolic activity of cells decreases with OGD duration, oppositely to

363

364

365

366

367

368

369

370

371

372

373

374

375

376

377

378

379

380

381

382

changes observed in nanomechanical measurements.

Based on the obtained results, we propose the following mechanism leading to cell deformability changes during OGD. The observed time-dependent decrease of Young's modulus in control, non-OGD treated SH-SY5Y cells was gradual regardless of the indentation depths chosen for the analysis (for low indentations of $400 \mathrm{~nm}$ and deeper indentations of $1200 \mathrm{~nm}$ ). Probably, it reflects the impact of glucose consumption on the mechanical properties of cells. As the dynamic of assembly and disassembly of F-actin is strongly dependent on the accessibility of adenosine triphosphate (ATP) molecules (ATP- G-actin binds to barbed end three times faster than GTP-G-actin, $\left.{ }^{46}\right)$, the reduction of ATP resulted in slow disassembly of cytoskeleton resulting in a gradual decrease of Young's modulus. A significantly lower level of ATP was reported to lead to cell softening 47 .

Several actin-associated proteins regulate actin polymerization/depolymerization as dynamic assembly and disassembly of the actin cytoskeleton is required for many biological processes, such as cell division, cell motility, endocytosis, and morphogenesis. These actin regulatory proteins contribute to nucleation, depolymerization, and fragmentation when a reorganization of the actin filaments is needed ${ }^{48-51}$. Cofilin is one of such proteins. It disassembles F-actin into tiny fragments of fibrous actin ${ }^{52}$. Although severing of F-actin by cofilin is independent of energy addition, the extensive reorganization of actin filaments demands high energy supplies 53 , in particular, to reach the level detected by AFM. It seems to be supported by the MTS assay showing unaltered metabolic activity of OGD-treated cells after one hour of 
385 the treatment. We postulate that in our measurements during the initial OGD, alterations

386 in mechanical properties of cells reflect the disassembling effect of cofilin on the cortical

387 actin. The effect is evident in cells after 1 hour of OGD. It is additionally detectable in

388 the experiments, in which changes in the effective surface area of a single cell are

389 quantified. The OGD duration of 1 hour is sufficient to induce a significant $(\sim 20 \%)$

390 reduction of the mean single cell surface area and increase the cell height. Such

391 increase suggested a more sparse actin scaffold and softening of the cells.

392 For longer OGD (3h and 12h), the cofilin-induced actin polymerization seems to be 393 attenuated as changes in nanomechanical properties of OGD-treated cells are less

394 pronounced. Severing and depolymerization of actin filaments by cofilin create many 395 small F-actin fragments with new barbed ends needed for their polymerisation ${ }^{54,55}$. 396 Simultaneously, after prolonged OGD time, cells are metabolically impaired as the MTS 397 assay reveals a significant drop in the number of metabolically active cells. It makes the 398 reorganization of the actin cortex less favorable, indicating that cofilin activity is inhibited 399 by phosphorylation of the serine residues at position 3 near the $\mathrm{N}$-end for longer OGD 400 duration. As a result, polymerization and stabilization of actin filaments are observed ${ }^{56}$. 401 Such effect leads to decreased cell deformability (cells become more rigid as Young's 402 modulus increases) in cells, in which metabolic activity is low. The latter affect the 403 rebuilding of the actin filaments network, which is low, as shown by small values of the 404 surface area of a single cell in cells after 3h and 12h of OGD.

406 mechanical properties did not fully recover. When comparing short-time OGD, the 407 elasticity of the cortical layer $(400 \mathrm{~nm})$ was well restored. The deep indentation, 
408 however, shows irreversible changes. Both shallow and deep indentations were

409 irreversible after $12 \mathrm{~h}$ OGD. The results contradict quantitative observation from actin

410 and tubulin in fluorescence microscopy, where any significant change can be clearly

411 observed. It indicates that changes in cell mechanics are more complex and cannot be

412 explained only by actin (de) polymerization. Actin rearrangement via activation of

413 profilin, cofilin and gelsolin, phosphorylation of myosin light chain, and changes in

414 membrane spectrin cytoskeleton might be involved ${ }^{57}$. It should also be noted that in

415 most studies, OGD constitutes the oxygen and glucose deprivations considered in

416 parallel. Separating the specific oxygen- and glucose-related contributions calls for

417 experiments conducted in conditions of either glucose or oxygen depletion ${ }^{58}$. The AFM-

418 derived nanomechanical properties of OGD and non-OGD treated cells reveal

419 a dominant role of glucose deprivation in 12 hours of OGD conditions that hinders the

420 oxygen depletion effect.

421 Numerous research that use the OGD model to understand mechanisms

422 involved in brain impairments ${ }^{59,60}$ has demonstrated that the pathological process of

423 ischemic stroke involves complex mechanisms acting on various cell types. These

424 studies focus on cell or molecular biology aspects. Cofilin, being involved in the dynamic

425 turnover of actin filaments, affects membrane integrity, receptor transport, and signal

426 transduction. Understanding mechanisms responsible for cofilin-related changes of

427 cytoskeleton remodeling, promise potential use of them to inhibit cofilin activity that

428 might induce neuroprotection through targeting diverse cellular components and

429 multiple pathways ${ }^{61,62}$. 


\section{Methods}

\section{Cell culture}

433 For experimental procedures, an undifferentiated $\mathrm{SH}-\mathrm{SY} 5 \mathrm{Y}$ human neuroblastoma cell

434 line was used. Cells were cultured in a Dulbecco's Modified Eagles' Medium (DMEM, 435 ATCC, LGC Standards) supplemented with 10\% Fetal Bovine Serum (FBS, ATCC, LGC 436 Standards). Cells were cultured in $35 \mathrm{~cm}^{2}$ culture flasks (TPP) and passaged $(<10)$ into 437 the corresponding plastic media required in each experiment. Cells were culture in the $438 \mathrm{CO}_{2}$ incubator (NUAIRE) at $37^{\circ} \mathrm{C}$ and $5 \% \mathrm{CO}_{2} / 95 \%$ air atmosphere.

\section{OGD experiments}

441 Cells were passaged from the culture flask to the Petri dish (TPP) and kept in the $\mathrm{CO}_{2}$ $442\left(37^{\circ} \mathrm{C}\right.$ and $5 \% \mathrm{CO}_{2} / 95 \%$ air atmosphere) for 24 hours in the DMEM (ATCC, LGC 443 Standards) supplemented with 10\% FBS. DMEM contains $4500 \mathrm{mg} / \mathrm{L}$ glucose and $4441 \mathrm{mM}$ sodium pyruvate. After this time, the medium was replaced either with (1) 445 neurobasal medium (NB) containing glucose (control cells, undergoing the same 446 treatment as OGD cells but without applying OGD conditions) or with neurobasal A 447 medium (NBA, without glucose used to create OGD conditions). NB is optimized for 448 prenatal and fetal neurons. NBA is optimized for growing postnatal and adult brain 449 neurons. These two media differ only in osmolality (260 mOsm versus 235 mOsm, 450 respectively).

451 OGD conditions were obtained in the following way. Cells were placed in the 452 temperature-controlled table $\mathrm{CO}_{2}$ incubator (Olympus) at $37^{\circ} \mathrm{C}$. The incubator was 453 connected to a gas exchange 3-input system (Tokai Hit) supplying air, $\mathrm{N}_{2}$, and $\mathrm{CO}_{2}$. In 
454 our system, $\mathrm{CO}_{2}$ concentration remained constant $(5 \%)$ while the air was replaced by

$455 \mathrm{~N}_{2}$, resulting in an oxygen concentration of $0.1 \%$. The oxygen level was maintained

456 constant by applying a gas flow at a level of $150 \mathrm{ml} / \mathrm{min}$. These parameters were kept

457 constant for 1, 3, and 12 hours. Immediately after applying OGD, cells were analyzed

458 using various techniques: MTS and LDH assays, atomic force microscopy (AFM),

459 epifluorescence, and confocal microscopy.

460

$461 \quad$ MTS assay

462 The viability and metabolic activity of SH-5YSY cells were verified by using an MTS

463 colorimetric test (Promega). Cells were cultured in 24-well plates in $1 \mathrm{ml}$ of the culture

464 medium (DMEM). Next, $100 \mu \mathrm{L}$ of MTS reagent (tetrazolium compound) was added to

465 the cells. Then, cells were incubated at $37 \circ \mathrm{C}$ in $95 \%$ air/5\% CO2 atmosphere in the

466 CO2 incubator (Nuaire) for $2 \mathrm{~h}$. The MTS method reduces tetrazolium compounds by

467 viable cells to generate a colored formazan product soluble in cell culture media. The

468 final volume of $1.1 \mathrm{~mL}$ was pipetted to 96 -well plates with $100 \mu \mathrm{L}$ per hole. The

469 absorbance $(O D=490 \mathrm{~nm})$ was recorded for $0 \mathrm{~h}$, $3 \mathrm{~h}$, and $24 \mathrm{~h}$ after OGD using a

470 spectrophotometer (ELISA SPECTROstar Nano, BMG LABTECH). The MTS assay was

471 repeated three times.

472

473 LDH assay

474 The cytotoxic effect of OGD was quantified by using CyQUANT TM LDH Cytotoxicity

475 Assay Kit (Invitrogen). Cells were plated on 24-well plates in $1 \mathrm{ml}$ of the corresponding

476 culture medium (Fig. 1). LDH level was evaluated in the following samples, i.e., (i) 
477 control and OGD cells, (ii) control and OGD cells treated with lysis buffer (10\% by

478 volume, $45 \mathrm{~min}$ in the $\mathrm{CO}_{2}$ incubator), (ii) culture medium (supernatant) taken from

479 control or OGD cells and separately treated with lysis buffer in an analogous way as (ii)

480 to verify how much cells detached during the medium exchange. Then, $50 \mu l$ of the

481 medium from each sample type was aspirated from each well and transferred into a 96-

482 well plate. Next, to each well, $50 \mu \mathrm{l}$ of the reaction mixture was added. After $1 \mathrm{~h}$ of

483 incubation in conditions protecting against light exposure, $50 \mu \mathrm{l}$ of stop solution was

484 added. Lactate dehydrogenase (LDH) is a cytosolic enzyme present in various cell

485 types. Damage of cell membrane results in a release of LDH to the surrounding

486 medium, which can be quantified by using LDH as a catalytic enzyme. It converts

487 lactate to pyruvate via NAD+ reduction to NADH. Oxidation of NADH by diphosphorase

488 reduces a tetrazolium salt to a red formazan product. OD at 490nm was registered

489 using an ELISA reader (Ledetect 96 ELISA, LED-based microplate reader, Labexim

490 Products) to detect it. The level of formazan is directly proportional to the level of LDH in

491 the surrounding medium. To obtain cell viability level the following equation was used:

$$
\%(\text { cell viability })=1-\frac{\text { experimental } L D H \text { release }\left(O D_{490}\right)}{\text { maximum } L D H \text { release }\left(O D_{490}\right)}
$$

where the maximum LDH release is the sum of LDH release in Triton $\mathrm{X} 100$ treated 496 samples (cells and supernatants of control and OGD-treated cells, respectively). 
499 To obtain changes in cofilin activity level, CytoGlow ${ }^{\mathrm{TM}}$ Cofilin (Phospho-Ser3)

500 Colorimetric Cell-Based ELISA Kit was applied (Assay Biotechnology) to monitor target

501 proteins concentration, here, in cells undergoing OGD treatment. Briefly, SH-SY5Y cells

502 (50,000 per well) were plated on a 96-well plate. After OGD experiments, cells were

503 fixed using 4\% paraformaldehyde and washed three times with $200 \mu$ l with Wash Buffer

504 (WB) for 5 minutes, each time gentle shook. Then, $100 \mu$ of quenching solution was

505 added for 20 minutes at room temperature (RT), followed by 3 times washing with WB

506 for 5 minutes at a time. Next, $200 \mu$ l of Blocking Buffer was added for 1 hour at RT, and,

507 afterwards, the plate was washed again ( $3 \times$ times, WB at RT). Then, a solution of $50 \mu l$

508 of each primary antibody against phosphorylated cofilin (Anti-Cofilin (Phospho-Ser3)

509 antibody), cofilin (Anti-cofilin antibody) and Glyceraldehyde 3-phosphate

510 dehydrogenase, GADPH (Anti-GAPDH antibody) was added to the corresponding well

511 and incubated for 16 hours (overnight) at $4^{\circ} \mathrm{C}$. Afterwards, they were rinsed 3 times with

$512200 \mu \mathrm{l}$ of WB for 5 minutes. In the next step, secondary antibodies (horseradish

513 peroxidase (HRP)-conjugated antiRabbit IgG antibody and/or HRP-conjugated anti-

514 Mouse IgG antibody) were added (50 $\mu \mathrm{l})$ for 1.5 incubation at RT. After incubation, the

515 plate with cells was washed, and $50 \mu \mathrm{l}$ of Ready-to-Use Substrate was added to each

516 well for 30 minutes at RT, followed by adding a Stop Solution. OD at $450 \mathrm{~nm}$ was

517 immediately read using a microplate reader (ELISA SPECTROstar Nano, BMG 518 LABTECH). 
522 The mechanical properties of cells were measured using AFM (CellHesion, Bruker-JPK,

523 Germany). The microscope is equipped with a constant temperature system. In our

524 experiments, the temperature was set to $32^{\circ} \mathrm{C}$ to provide the cell survival conditions and

525 the cantilever stability. Cells were indented with silicon nitride cantilevers (ORC-8,

526 Bruker) characterized by a nominal spring constant of $0.03 \mathrm{~N} / \mathrm{m}$ and an open half-angle

527 of $36^{\circ}$. All measurements were conducted in a force spectroscopy mode. The spring

528 constants of used cantilevers were determined using the Sader method ${ }^{63}$. The average

529 value was $0.058 \pm 0.005$ for $n=8$ cantilevers. A force map of 6 per 6 pixels

530 (corresponding to a $6 \mu \mathrm{m} \times 6 \mu \mathrm{m}$ scan size) was recorded on each cell.

531 The force curves (i.e. the dependence of the cantilever deflection recorded as a function

532 of relative sample position) were acquired at the approach/retract velocity of $8 \mu \mathrm{m} / \mathrm{s}$. On

533 individual plastic Petri dishes, two groups of force curves were collected. First,

534 calibration curves were acquired on a Petri dish bottom surface (a reference calibration

535 curve). Next, force curves were recorded on living cells. Next, force curves were

536 recorded on living cells. Force curves were collected by setting a grid of $6 \times 6$ points

537 that corresponded to $6 \mu \mathrm{m} \times 6 \mu \mathrm{m}$ scan area. A grid was set over the nuclear region to

538 minimize the influence of the underlying stiff plastic surface. All measurements were

539 conducted in DMEM and were repeated 3 times.

\section{Young's modulus determination}

542 The subtraction of the calibration curves from a curve collected on a living cell produces

543 the relation between load force and indentations depth. This relation was analyzed 
544 using the Hertz-Sneddon contact mechanics. The AFM probe was approximated by the cone that resulted in the following relation between load force and indentation depth:

$$
F(\delta)=\frac{2 \cdot \tan (\kappa)}{\pi} \times \frac{E_{c e l l}}{1-\mu^{2}} \cdot \delta^{2}
$$

where $F$ is load force, $\delta$ is the indentation depth, Ecell is the apparent Young's modulus

550 of the cell, and $\mu$ is the Poisson's ratio (equalled to 0.5 assuming that cells are 551 incompressible materials). The final modulus value was expressed as a mean and 552 standard deviation from all measured cells.

\section{Fluorescence (epifluorescence and confocal) microscopy}

555 Visualization of actin filaments, microtubules, and cell nuclei was performed by using and 40x magnification, $100 \mathrm{~W}$ mercury lamp (illuminating the whole cell area uniformly), using Orca Spark digital camera providing a 2.3 megapixel (1920x1200) pixel image and analyzed with ImageJ (ImageJ 1.53e https://imagej.nih.gov/ij/). Cells cultured on

562 phosphate-buffered saline (PBS, Sigma), treated with a cold $0.2 \%$ Triton X-100 solution,

564 antibody conjugated with Cy3 for 24 hours. The next day, samples were stained with stained by 10 min incubation with Hoechst dye. 
567 Confocal images of actin and microtubular cytoskeleton were recorded at the

568 Laboratory of in vivo and in vitro Imaging (Maj Institute of Pharmacology Polish

569 Academy of Science, Cracow, Poland). They were recorded using a Leica TCS SP8

570 WLL confocal microscope equipped with new-generation HyD detectors set at 415-450

$571 \mathrm{~nm}$ (Hoechst) and 509-560 nm (Alexa Fluor 488). Fluorescent dyes were excited by

572 diode lasers: $405 \mathrm{~nm}$ (Hoechst) and white light laser with emission wavelength set at $573499 \mathrm{~nm}$ (AlexaFluor 488). Images were registered using an oil immersion 63x objective 574 lens (HC PL APO CS2 NA 1.40).

575

576 Surface area determination

577 A single cell effective surface area (SA) was applied to characterize how well cells 578 spread on the surface at given conditions. This value describes an average surface 579 area occupied by an individual cell. Images of fluorescently stained cells (F-actin using 580 phalloidin-Alexa Fluor 488 dye, cell nuclei by Hoechst 33342) were binarised using 581 ImageJ software. From these images, the surface area occupied by cells was 582 determined. Next, cell nuclei were manually counted to receive the number of cells. 583 Finally, the surface area occupied by cells was divided by the number of cells that 584 enabled the calculation of the effective surface area of a single cell. Images were 585 acquired during three repetitive experiments, which resulted in 20 images per condition 586 to be analyzed. The total number of cells was at least 8000 cells. 
590 Nucleus - to - cytoplasm (N/C ratio)

591 To obtain the N/C, the effective area of individual cell nuclei was quantified analogously

592 as the effective surface area of a single cell was determined. Next, the effective surface 593 area of a single nucleus was divided by the effective surface area of a single cell. The 594 total number of images analyzed was 20 per condition.

595

\section{Statistical analysis}

597 All data are presented as the mean \pm standard deviation from $\mathrm{n}$ repetitions. In all 598 figures, box plots were applied to show the basic statistical descriptors: mean (open 599 square), median (black line), standard deviation (whiskers), and 25\% and $75 \%$ 600 percentiles (box). Statistical significance was verified by applying the non-parametric 601 Mann-Whitney test (Origin 9.2 Pro). Significance is indicated by $p$ values (ns - not 602 statistically different, $\left.p>0.05 ;{ }^{*} p<0.05,{ }^{* *} p<0.01,{ }^{* *} p<0.001\right)$.

604 Acknowledgments:

605 TZ acknowledges the support of project no. POWR.03.02.00-00-1013/16 (InterDokMed). 606 The APC was funded by project no. POWR.03.02.00-00-1013/16 (InterDokMed)". The 607 authors are thankful to prof. Danuta Jantas and prof. Halina Jurkowska for sharing the 608 SH-SY5Y cell lines.

609

610 Author Contributions:

611 Conceptualization, TZ, ML, JP and BZ; methodology, TZ and ML; validation, TZ, BZ, JP 612 and ML; formal analysis, TZ; investigation, TZ; resources, ML; data curation, TZ; writing 
613 - original draft preparation, TZ, BZ, JP, ML; writing —review and editing TZ, BZ, JP, ML;

614 confocal images visualization, JW; cell cultures, JP; supervision, ML and JP; funding

615 acquisition, ML; All authors have read and agreed to the published version of the

616 manuscript.

617

618 Data Availability Statement:

619 Correspondence and requests for materials should be addressed to ML.

620

621 Competing interests

622 The authors declare no competing interests

623

624 
625

626

627

628

629

630

631

632

633

634

635

636

637

638

639

640

641

642

643

644

645

646

647

648

649

650

651

652

653

\section{FIGURE CAPTIONS:}

Figure 1. (a) A scheme showing three steps of sequential OGD applied to living SHSY5Y cells. Firstly, cells were cultured for 24 hours after seeding in $5 \% \mathrm{CO}_{2}, 95 \%$ atmosphere $\left(37^{\circ} \mathrm{C}\right)$ in a DMEM with $4500 \mathrm{mg} / \mathrm{ml}$ of glucose $(\operatorname{DMEM}(+\mathrm{G}))$. They refer here as control cells. Next, the medium was exchanged to NBA(-G), and cells were placed in a table $\mathrm{CO}_{2}$ incubator for $1 \mathrm{~h}, 3 \mathrm{~h}$, or $12 \mathrm{~h}$ at $0.1 \% \mathrm{O}_{2}$ (referred to as OGD conditions and OGD cells). Finally, OGD cells were rinsed with a $D M E M(+G)$ in the atmosphere of $5 \% \mathrm{CO}_{2}$ and $95 \%$ air (reoxygenation conditions, , in addition, non-OGD cells were kept in $\operatorname{DMEM}(+\mathrm{G})$ ). (b) Phase-contrast image showing the morphology of neuroblastoma SH-SY5Y cells cultured for $24 \mathrm{~h}$ in $\mathrm{NB}(+\mathrm{G})$, as it induced differentiation resulting in a neuron-like morphology with numerous, fine protrusions (neurite-like structures). Scale bar $-50 \mu \mathrm{m}$.

Figure 2. Metabolic level and viability of SH-SY5Y neuroblastoma cells assessed by MTS (a,b) and LDH (c,d) assays, directly after OGD (a,c) and after 24h of reoxygenation $(b, d)$. Each dot denotes a single readout from the ELISA reader. $(a, b) A$ mean (open circle), median (black line), standard deviation (SD, box size) were determined from data gathered from 3 independent repetitions. (c,d) Columns represent a mean value from 12 ELISA readouts ( $n=3$ independent repetitions). Relative absorbance was normalized to values obtained for the control samples. Statistical significance: $n s-$ not statistically significant, $p>0.05,{ }^{*} p<0.05,{ }^{* *} p<0.001$.

Figure 3. Nanomechanical properties of SH-SY5Y neuroblastoma cells after OGD treatment, quantified by the apparent Young's modulus calculated for the indentation depth of $400 \mathrm{~nm}$ (a) and $1200 \mathrm{~nm}$ (b). Four groups of cells were compared: control (C, $\mathrm{NB}(+\mathrm{G})$ ), OGD cells (OGD 1h, 3h, or 12h, NBA(-G)), reoxygenated OGD cells (RO 24h, $\operatorname{DMEM}(+G)$ ), and control, non-OGD cells $(C 24 \mathrm{~h})$ kept in $\operatorname{DMEM}(+G)$ for the same time as reoxygenated OGD cells. Box plots represent a median (black line), a mean (solid square), standard deviation (whiskers), and $25 \%$ and $75 \%$ percentiles (box) from $n=60$ 
654 cells. Statistical significance: ns - not statistically significant $(p>0.05),{ }^{*} p<0.05$, $655{ }^{* *} p<0.01,{ }^{* * *} p<0.001$.

656

657 Figure 4. Confocal images of the actin cytoskeleton in OGD-treated and reoxygenated 658 cells. Staining: actin filaments - phalloidin conjugated with Alexa Fluor 488, cell nuclei 659 Hoechst 33342; scale bar $25 \mu \mathrm{m}$.

660

661 Figure 5. Spreading area (SA) of cells after OGD (a) and reoxygenation (b). Each dot 662 denotes an average surface area of individual cells. Boxplot represents basic statistical 663 parameters (mean, median, standard deviation, and 25\% and $75 \%$ percentiles from $664 n=60$ fluorescent images). Statistical significance: $n s$ - not statistically significant 665 $(p>0.05),{ }^{*} p<0.05,{ }^{* *} p<0.001$.

666

667

Figure 6. Nucleus to cytoplasm $(N / C)$ ratio of cells after OGD treatment and 668 reoxygenation. Each dot denotes an average value of individual cells. Boxplot 669 represents basic statistical parameters, i.e., mean (open square), median (line), and

670 standard deviation, from $n=60$ cells. Statistical significance: $n s$ - not statistically 671 significant ${ }^{*} p<0.05,{ }^{* *} p<0.001$.

672

673 Figure 7. Cofilin and p-cofilin (phosphorylated cofilin) expression level in SH-SY5Y cells 674 upon 1h (a) and 12h (b) OGD. Control cells were kept in NB(+G), while OGD cells were 675 kept in $\operatorname{NBA}(-\mathrm{G})$. A mean (black square), median (black middle line), standard deviation 676 (SD, outside black lines) were determined from data gathered from 3 independent 677 repetitions. Statistical significance $\left(n s-\right.$ not statistically significant, ${ }^{* *} p<0.01,{ }^{* *} p<$ $6780.001)$. 
679

680

681

682

683

684

685

686

687

688

689

690

691

692

693

694

695

696

697

698

699

700

701

\section{References}

1. Katan, M. \& Luft, A. Global Burden of Stroke. Semin. Neurol. 38, 208-211 (2018).

2. Sennfält, S., Norrving, B., Petersson, J. \& Ullberg, T. Long-Term Survival and Function after Stroke: A Longitudinal Observational Study from the Swedish Stroke Register. Stroke 50, 53-61 (2019).

3. Tasca, C. I., Dal-Cim, T. \& Cimarosti, H. In vitro oxygen-glucose deprivation to study ischemic cell death. Methods Mol. Biol. 1254, 197-210 (2015).

4. Kalogeris, T., Baines, C. P., Krenz, M. \& Korthuis, R. J. Cell biology of ischemia/reperfusion injury. Int. Rev. Cell Mol. Biol. 298, 229-317 (2012).

5. Shi, Y. et al. Rapid endothelial cytoskeletal reorganization enables early bloodbrain barrier disruption and long-term ischaemic reperfusion brain injury. Nat. Commun. 7, 10523 (2016).

6. Wang, R. et al. Oxygen-Glucose deprivation induced Glial scar-like change in Astrocytes. PLoS One 7, e37574 (2012).

7. Spence, E. F. \& Soderling, S. H. Actin out: Regulation of the synaptic cytoskeleton. J. Biol. Chem. 290, 28613-28622 (2015).

8. Etienne-Manneville, S. Actin and microtubules in cell motility: Which one is in control? Traffic 5, 470-477 (2004).

9. Schoumacher, M., Goldman, R. D., Louvard, D. \& Vignjevic, D. M. Actin, microtubules, and vimentin intermediate filaments cooperate for elongation of invadopodia. J. Cell Biol. 189, 541-556 (2010).

10. Hohmann \& Dehghani. The Cytoskeleton-A Complex Interacting Meshwork. Cells 8, 362 (2019). 
702

703

704

705

706

707

708

709

710

711

712

713

714

715

716

717

718

719

720

721

722

723

724

11. Kristó, I., Bajusz, I., Bajusz, C., Borkúti, P. \& Vilmos, P. Actin, actin-binding proteins, and actin-related proteins in the nucleus. Histochem. Cell Biol. 145, 373-388 (2016).

12. Tilve, S., Difato, F. \& Chieregatti, E. Cofilin 1 activation prevents the defects in axon elongation and guidance induced by extracellular alpha-synuclein. Sci. Rep.

5, 16524 (2015).

13. Bamburg, J. R., Minamide, L. S., Wiggan, O., Tahtamouni, L. H. \& Kuhn, T. B. Cofilin and actin dynamics: Multiple modes of regulation and their impacts in neuronal development and degeneration. Cells 10, 2726 (2021).

14. Suurna, M. V. et al. Cofilin mediates ATP depletion-induced endothelial cell actin alterations. Am. J. Physiol. - Ren. Physiol. 290, F1398-F1407 (2006).

15. Chen, B. et al. Cofilin Inhibition by Limk1 Reduces Rod Formation and Cell Apoptosis after Ischemic Stroke. Neuroscience 444, 64-75 (2020).

16. Wiggan, O., Shaw, A. E., DeLuca, J. G. \& Bamburg, J. R. ADF/Cofilin Regulates Actomyosin Assembly through Competitive Inhibition of Myosin II Binding to FActin. Dev. Cell 22, 530-543 (2012).

17. Liu, Y. et al. Human ischaemic cascade studies using SH-SY5Y cells: A systematic review and meta-analysis. Transl. Stroke Res. 9, 564-574 (2018).

18. Kovalevich, J. \& Langford, D. Considerations for the use of SH-SY5Y neuroblastoma cells in neurobiology. Methods Mol. Biol. 1078, 9-21 (2013).

19. Lopes, F. M. et al. Comparison between proliferative and neuron-like SH-SY5Y cells as an in vitro model for Parkinson disease studies. Brain Res. 1337, 85-94 (2010). 
725

726

727

728

729

730

731

732

733

734

735

736

737

738

739

740

20. Appukuttan, T. A. et al. Parkinson's disease cybrids, differentiated or undifferentiated, maintain morphological and biochemical phenotypes different from those of control cybrids. J. Neurosci. Res. 91, 963-970 (2013).

21. Binnig, G., Quate, C. F. \& Gerber, C. Atomic force microscope. Phys. Rev. Lett. 56, 930-933 (1986).

22. Lekka, M. Discrimination Between Normal and Cancerous Cells Using AFM. Bionanoscience 6, 65-80 (2016).

23. Pyka-Fościak, G., Zemła, J., Lis, G. J. J., Litwin, J. A. A. \& Lekka, M. Changes in spinal cord stiffness in the course of experimental autoimmune encephalomyelitis, a mouse model of multiple sclerosis. Arch. Biochem. Biophys. 680, 108221 (2020).

24. Ciasca, G. et al. Nanomechanical signature of brain tumours. Nanoscale 8, 19629-19643 (2016).

25. Ciasca, G. et al. Mapping viscoelastic properties of healthy and pathological red blood cells at the nanoscale level. Nanoscale 7, 17030-17037 (2015).

26. Fang, Y. et al. Investigating dynamic structural and mechanical changes of neuroblastoma cells associated with glutamate-mediated neurodegeneration. Sci. Rep. 4, 7074 (2014).

27. Kubiak, A. et al. Stiffening of DU145 prostate cancer cells driven by actin filaments-microtubule crosstalk conferring resistance to microtubule-targeting drugs. Nanoscale 13, 6212-6226 (2021).

28. Gostek, J. et al. Nano-characterization of two closely related melanoma cell lines with different metastatic potential. Eur. Biophys. J. 44, 49-55 (2015). 
29. Scudiero, D. A. et al. Evaluation of a Soluble Tetrazolium/Formazan Assay for Cell Growth and Drug Sensitivity in Culture Using Human and Other Tumor Cell Lines. Cancer Res. 48, 4827-4833 (1988).

30. Lee, W. T. et al. Neuroprotective effects of agmatine on oxygen-glucose deprived primary-cultured astrocytes and nuclear translocation of nuclear factor-kappa B. Brain Res. 1281, 64-70 (2009).

31. Yang, Y. \& Sauve, A. A. NAD(+) metabolism: Bioenergetics, signaling and manipulation for therapy. Biochim. Biophys. Acta 1864, 1787-1800 (2016).

32. Liu, H. B. et al. The effects of ABCG2 on the viability, proliferation and paracrine actions of kidney side population cells under oxygen-glucose deprivation. Int. J. Med. Sci. 11, 1001-1008 (2014).

33. Chighizola, M., Puricelli, L., Bellon, L. \& Podestà, A. Large colloidal probes for atomic force microscopy: Fabrication and calibration issues. J. Mol. Recognit. 34, e2879 (2021).

34. Sneddon, I. N. The relation between load and penetration in the axisymmetric boussinesq problem for a punch of arbitrary profile. Int. J. Eng. Sci. 3, 47-57 (1965).

35. Fujii, Y. et al. Spatiotemporal dynamics of single cell stiffness in the early developing ascidian chordate embryo. Commun. Biol. 4, 341 (2021).

36. Chighizola, M. et al. Adhesion force spectroscopy with nanostructured colloidal probes reveals nanotopography-dependent early mechanotransductive interactions at the cell membrane level. Nanoscale 12, 14708-14723 (2020).

37. Bernstein, B. W. \& Bamburg, J. R. ADF/Cofilin: A functional node in cell biology. 
Trends Cell Biol. 20, 187-195 (2010).

772

773

774

775

776

777

778

779

780

781

782

783

784

785

786

787

788

789

790

791

792

38. Bravo-Cordero, J. J., Magalhaes, M. A. O., Eddy, R. J., Hodgson, L. \& Condeelis, J. Functions of cofilin in cell locomotion and invasion. Nat. Rev. Mol. Cell Biol. 14, 405-417 (2013).

39. Bamburg, J. R. \& Bernstein, B. W. Roles of ADF/cofilin in actin polymerization and beyond. F1000 Biol. Rep. 2, 62 (2010).

40. Kleman, A. M., Yuan, J. Y., Aja, S., Ronnett, G. V. \& Landree, L. E. Physiological glucose is critical for optimized neuronal viability and AMPK responsiveness in vitro. J. Neurosci. Methods 167, 292-301 (2008).

41. Cross, J. L., Meloni, B. P., Bakker, A. J., Lee, S. \& Knuckey, N. W. Modes of neuronal calcium entry and homeostasis following cerebral ischemia. Stroke Res. Treat. 2010, 316862 (2010).

42. Carinci, M. et al. Different roles of mitochondria in cell death and inflammation: Focusing on mitochondrial quality control in ischemic stroke and reperfusion. Biomedicines 9, 169 (2021).

43. Chen, $\mathrm{H}$. et al. Oxidative stress in ischemic brain damage: Mechanisms of cell death and potential molecular targets for neuroprotection. Antioxid. Redox Signal. 14, 1505-1517 (2011).

44. Michalski, D. et al. A novel approach for mechanical tissue characterization indicates decreased elastic strength in brain areas affected by experimental thromboembolic stroke. Neuroreport 26, 583-587 (2015).

45. Discher, D. E., Janmey, P. \& Wang, Y. L. Tissue cells feel and respond to the stiffness of their substrate. Science 310, 1139-1143 (2005). 
794 46. Korn, E. D., Carlier, M. F. \& Pantaloni, D. Actin polymerization and ATP

795 hydrolysis. Science 238, 638-644 (1987).

796

797

798

799

800

801

802

803

804

805

806

807

808

809

810

811

812

813

814

815

47. Aronoff, S. L., Berkowitz, K., Shreiner, B. \& Want, L. Glucose Metabolism and Regulation: Beyond Insulin and Glucagon. Diabetes Spectr. 17, 183-190 (2004).

48. Pollard, T. D., Blanchoin, L. \& Mullins, R. D. Molecular mechanisms controlling actin filament dynamics in nonmuscle cells. Annu. Rev. Biophys. Biomol. Struct. 29, 545-576 (2000).

49. Brunello, E. et al. Myosin filament-based regulation of the dynamics of contraction in heart muscle. Proc. Natl. Acad. Sci. 117, 8177-8186 (2020).

50. Schaks, M., Giannone, G. \& Rottner, K. Actin dynamics in cell migration. Essays Biochem. 63, 483-495 (2019).

51. Le, S., Yu, M., Bershadsky, A. \& Yan, J. Mechanical regulation of formindependent actin polymerization. Sem. Cell Develop. Biol. 102, 73-80 (2020).

52. Condeelis, J. How is actin polymerization nucleated in vivo? Trends Cell Biol. 11, 288-293 (2001).

53. Kuiper, J. W. P. et al. Creatine kinase-mediated ATP supply fuels actin-based events in phagocytosis. PLOS Biol. 6, 0568-0580 (2008).

54. Papakonstanti, E. A. \& Stournaras, C. Cell responses regulated by early reorganization of actin cytoskeleton. FEBS Letters 582, 2120-2127 (2008).

55. Oser, M. \& Condeelis, J. The cofilin activity cycle in lamellipodia and invadopodia. J. Cell. Biochem. 108, 1252-1262 (2009).

56. Moriyama, K., lida, K. \& Yahara, I. Phosphorylation of Ser-3 of cofilin regulates its essential function on actin. Genes Cells 1, 73-86 (1996). 
817 57. Li, H. et al. Cytoskeleton Remodeling Induces Membrane Stiffness and Stability Changes of Maturing Reticulocytes. Biophys. J. 114, 2014-2023 (2018).

58. Mathews, E. H., Stander, B. A., Joubert, A. M. \& Liebenberg, L. Tumor cell culture survival following glucose and glutamine deprivation at typical physiological concentrations. Nutrition 30, 218-227 (2014).

822 59. Hoshi, Y. et al. Ischemic brain injury leads to brain edema via hyperthermiainduced TRPV4 activation. J. Neurosci. 38, 5700-5709 (2018).

824 60. Tornabene, E., Helms, H. C. C., Pedersen, S. F. \& Brodin, B. Effects of oxygen825 glucose deprivation (OGD) on barrier properties and mRNA transcript levels of selected marker proteins in brain endothelial cells/astrocyte co-cultures. PLoS One 14, e0221103 (2019).

61. Alhabidi, Q., Sayeed, M.S.B., Shah, Z.A. Cofilin as a Promising Therapeutic Target for Ischemic and Hemorrhagic Stroke. Transl. Stroke Res. 7, 33-41 (2016). of Cofilin-1 in the Motor Cortex. Front. Cell Dev. Biol. 9, 634347 (2021).

832 63. Sader, J. E., Larson, I., Mulvaney, P. \& White, L. R. Method for the calibration of atomic force microscope cantilevers. Rev. Sci. Instrum. 66, 3789-3798 (1995). 